\title{
Oligodendroglia: metabolic supporters of neurons
}

\author{
Thomas Philips and Jeffrey D. Rothstein \\ Brain Science Institute and Department of Neurology, Johns Hopkins University, Baltimore, Maryland, USA.
}

\begin{abstract}
Oligodendrocytes are glial cells that populate the entire CNS after they have differentiated from oligodendrocyte progenitor cells. From birth onward, oligodendrocytes initiate wrapping of neuronal axons with a multilamellar lipid structure called myelin. Apart from their well-established function in action potential propagation, more recent data indicate that oligodendrocytes are essential for providing metabolic support to neurons. Oligodendrocytes transfer energy metabolites to neurons through cytoplasmic "myelinic" channels and monocarboxylate transporters, which allow for the fast delivery of short-carbon-chain energy metabolites like pyruvate and lactate to neurons. These substrates are metabolized and contribute to ATP synthesis in neurons. This Review will discuss our current understanding of this metabolic supportive function of oligodendrocytes and its potential impact in human neurodegenerative disease and related animal models.
\end{abstract}

\section{Introduction}

Oligodendrocytes are the cells in the CNS that produce myelin, a multilamellar lipid structure that wraps around axons and ensures proper conduction of action potentials. Oligodendrocytes are derived from oligodendrocyte progenitor cells (OPCs, also called NG2-glia), which populate the CNS starting from embryonic development onward (1-3). In mice, OPCs continue to give rise to new oligodendrocytes; after P30, oligodendrogenesis progressively slows down as the animals age $(4,5)$. The oligodendrocyteproduced myelin sheath is classically characterized by the existence of multiple myelin segments along the axon separated by regions devoid of myelin called nodes of Ranvier (for recent reviews see refs. 6-8). Unlike their peripheral analog the Schwann cell, a single oligodendrocyte can produce multiple myelin segments on multiple axons. Once myelination is completed, adultborn oligodendrocytes continue to contribute to myelination and myelin remodeling (9-11). Not every axon is fully myelinated along its length, and large stretches are unmyelinated. A recent study investigated myelination in mouse neocortex using highthroughput reconstruction of electron microscopic images, which identified three distinct profiles of myelin distribution along the axons of pyramidal neurons in mouse neocortex (12): unmyelinated axons, intermittently myelinated axons, and axons with a very long unmyelinated segment preceding the first myelin internode. The true role of these different profiles in CNS communication remains to be investigated, but it is suggested that they allow for highly complex neuronal behavior through different arrays of communication mechanisms. Many cell-intrinsic and -extrinsic pathways may contribute to oligodendrocyte myelination and are reviewed elsewhere $(8,13,14)$. The mechanism by which these myelin layers wrap around an axon has recently been elucidated using an elegant approach combining live in vivo imaging in zebrafish with electron microscopy. Newly generated myelin layers were found to be deposited by continuous spiraling of the

Conflict of interest: The authors have declared that no conflict of interest exists. Reference information: J Clin Invest. 2017;127(9):3271-3280.

https://doi.org/10.1172/JCI90610. innermost "tongue" of the myelin around the axon, with simultaneous lateral myelin spread along the axonal length (15). This process is dependent on dynamic changes in actin assembly (16, 17). One of the main proteins expressed in the myelin, myelin basic protein (MBP), drives myelin actin disassembly and myelin compaction $(16,18)$, whereas another oligodendrocyte protein, 2',3'-cyclic-nucleotide 3'-phosphodiesterase (CNPase), counteracts the myelin compaction by promoting actin assembly (19). Actin assembly allows for uncompacted myelinic channels to develop within the myelin layers, which in turn promotes transfer of small molecules and metabolites between different regions within the oligodendrocyte cytoplasm (see below and ref. 19).

Multiple studies have shown that myelin deposition is not static, but far more dynamic than initially thought, with adaptive changes in myelin driven by changes in neuronal activity (myelin plasticity) $(6,20-25)$. These changes, e.g., induced by optogenetic stimulation or stimulated through learning of complex motor skills, promote oligodendrogenesis and the deposition of new myelin sheaths along the axons. In one study, blocking oligodendrogenesis by deleting the oligodendrocyte transcription factor myelin regulatory factor (Myrf) inhibited de novo myelination, and mice subsequently failed to learn a motor task, suggesting that new myelin synthesis and deposition along neurons are crucial for learning complex motor skills (21). Adaptive changes in myelin in human brain have also been suggested by several studies $(26,27)$. Learning a second language or training a visuomotor skill induced changes in white matter structure as found with diffusing tensor imaging. Nevertheless, whether changes in white matter structure truly reflect adaptive changes in oligodendrogenesis and myelination remains to be further clarified.

\section{Oligodendrocytes provide metabolic support to neurons}

Neurons have significant metabolic needs. Axons in human adults are often very long (sometimes more than $1 \mathrm{~m}$ ) and represent a huge burden on the neuron's limited energy storage and supply; therefore, it is not surprising that axons are particularly vulnerable in conditions of increased electrical activity. These energy 


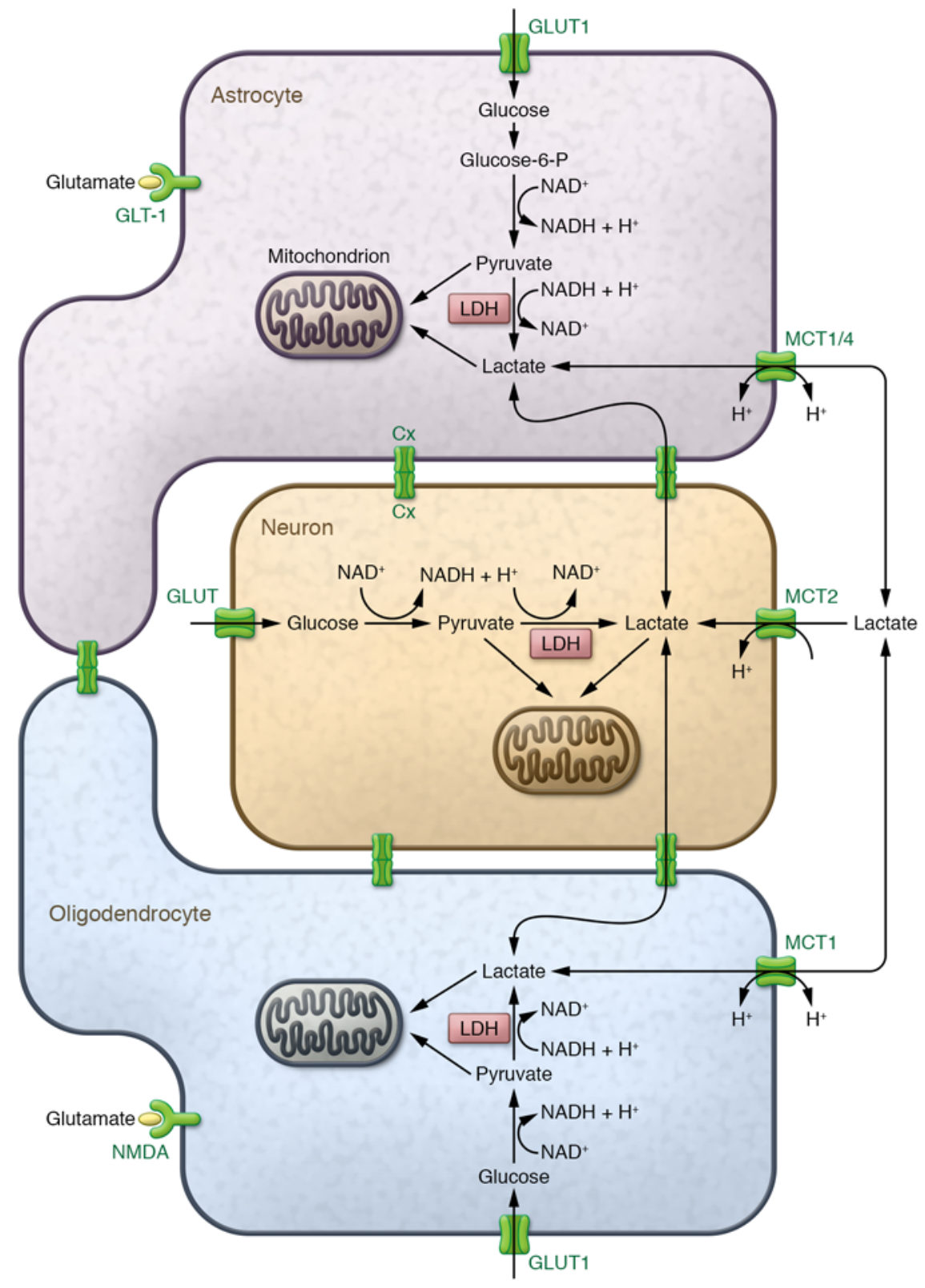

Figure 1. Astrocyte-oligodendrocyte-neuron lactate shuttle. Astrocytes (top) can take up glucose from the blood circulation through glucose transporters (GLUT1), a process that is enhanced upon glutamate release from neurons and glutamate binding to astrocyte-expressed glutamate transporters (GLT-1). Glycolysis breaks down glucose to pyruvate with the production of ATP and NADH. Alternatively, astrocytes can break down intracellular glucose, which is stored as glycogen. Subsequently, pyruvate is either metabolized in the mitochondria (leading to additional production of ATP) or converted to lactate by lactate dehydrogenase (LDH) when oxygen is limited (ensuring the continuation of glycolysis through oxidation of $\mathrm{NADH}$ to $\mathrm{NAD}^{+}$ to resupply the $N A D^{+}$pool). In oligodendrocytes (bottom), glucose can similarly be processed into pyruvate and lactate. Oligodendrocyte glucose uptake is controlled by glutamate binding to the NMDA receptor expressed on the oligodendrocyte surface. Intracellular lactate in astrocytes or oligodendrocytes can either exit the cell through the monocarboxylate transporters MCT1 and MCT4 or be converted back to pyruvate in the mitochondria, where it fuels ATP or fatty acid synthesis. Extracellular lactate can be shuttled to neurons through neuronal MCT2 and used to fuel neuronal ATP synthesis in the mitochondria. Gap junction coupling $(C x)$ between glial cells and glial cells/neurons mediates the exchange of metabolites like pyruvate and lactate, but the importance of these connections in metabolic shuttling is still to be further explored. Alternatively, it is hypothesized that extracellular lactate is imported in glial cells and could contribute to ATP synthesis in glial cells upon conversion to pyruvate in the mitochondria. demands could be met by metabolism of glucose by glycolysis, followed by entry of pyruvate into the mitochondrial TCA cycle and oxidative phosphorylation, generating ATP for the neuron (Figure 1). Neurons do not have significant energy stores $(28,29)$ (as compared with astrocytes, which store glucose as glycogen), and require significant amounts of ATP to maintain the activity of the $\mathrm{Na}^{+} / \mathrm{K}^{+}$channels, ensuring the continuation of repetitive firing of action potentials. $\mathrm{Na}^{+} / \mathrm{K}^{+}$channels are expressed along the internodes, where most of the axonal mitochondria are located, shielded from the extracellular environment by the myelin sheath $(30,31)$. Therefore, it would not be surprising if oligodendrocytes have undergone evolutionary adaptation to neuronal energy demands (Figure 2).

The concept that glial cells contribute to neuronal metabolic support was proposed several decades ago with the so-called astrocyte-neuron lactate shuttle hypothesis (refs. 32, 33, Figure 1, Figure 3i, and Figure 3ii), which principally focuses on neuron- astrocyte interactions. Upon glutamate release from electrically active neurons, astrocyte glutamate transporter activity drives astrocyte glucose uptake and subsequent conversion of glucose into pyruvate and lactate in these cells (Figure 1 and Figure 3i). These metabolites can be shuttled toward neurons through monocarboxylate transporters (discussed below) to provide neurons with metabolic support. Similarly, an increase in ammonium $\left(\mathrm{NH}_{4}^{+}\right)$release upon increased neuronal activity can increase lactate levels in astrocytes by promoting pyruvate to lactate conversion followed by lactate release (34). Upon neuronal entry, lactate can be converted into pyruvate by neuronal lactate dehydrogenase and can enter the TCA cycle to fuel neuronal energy homeostasis (Figure 1). Astrocyte-derived lactate has indeed been shown to be an essential contributor to neuronal metabolism (see below); however, astrocyte contact with neurons is generally spatially limited to the neuronal cell body, the regions of synaptic contact, and some nodes of Ranvier. In contrast, oligodendrocytes are well 


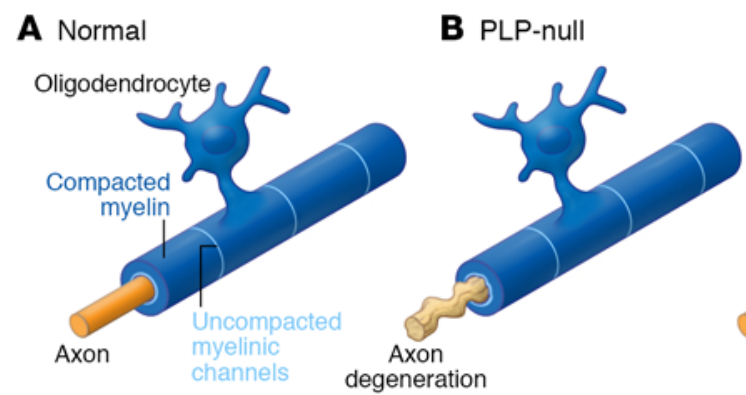

C MBP-null

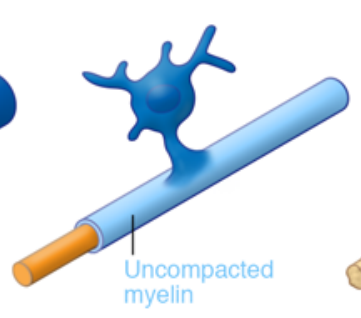

D CNPase-null

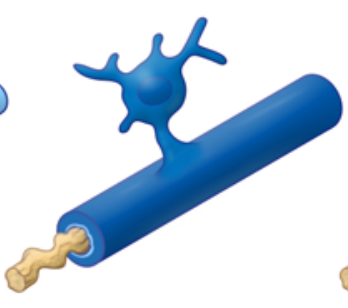

E MCT1-null

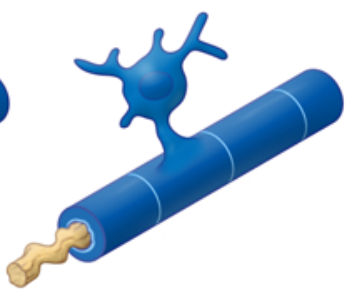

Figure 2. Oligodendrocytes provide metabolic support to neurons. (A) Normal myelin with compacted (dark blue) and uncompacted (light blue) channels form a multilamellar structure around the axon. (B) In PLP-null animals, myelin appears to be ultrastructurally normal, but the underlying axons show signs of degeneration. (C) In MBP-null mice (shiverer mice), only a thin sheath of uncompacted myelin wraps the axon. Despite this dysmyelination, these mice develop normally and axons are intact. (D) The myelin in CNPase-null mice is more compacted than normal as a result of the loss of the uncompacted myelinic channels, resulting in severe axonal degeneration. (E) Loss of MCT1 in oligodendrocytes leads to axonal degeneration despite normal-appearing myelin wrapping the axons. All these models show a clear distinction between the ability of myelin to support axonal conductance and its ability to provide trophic support to the axon. How myelinic channels are affected in the PLP- and MCT1-null mice remains to be elucidated.

connected to the axon through the myelin sheath, perfectly poised to support the metabolic demands of neurons.

Oligodendrocyte neuronal metabolic support. The first studies indicating a metabolic role for oligodendrocytes were knockout studies of specific oligodendrocyte proteins expressed in noncompacted or compacted myelin (Figure 2). Loss of the oligodendrocyte-specific myelin proteolipid protein (PLP) in transgenic animals causes severe axonal degeneration in the absence of alterations in the electrical conduction properties of the axon (refs. 35, 36, and Figure 2B). This would suggest that myelinated axons need oligodendrocyte metabolic support to maintain ATP homeostasis. The dysmyelinating mutant "shiverer" mouse, which lacks the oligodendrocyte protein MBP, does not show any signs of axonal degeneration except for mild axonal swellings in the cerebellar granular cell layer and spinal cord white matter (37, 38 ), despite the absence of compacted myelin and abrogation of normal action potential propagation (ref. 39 and Figure 2C). These animals develop severe tremor and seizure-like behavior and die around 3 months of age. Despite the inability of the myelin to support electrical activity of the neurons in these mice, axons do not degenerate, perhaps because of ongoing metabolic support from the thin, uncompacted myelin process (35). Notably, the phenotype observed in these MBP- and PLP-null mice clearly suggests that oligodendrocytes affect neuronal homeostasis independent of their capacity to provide normal axonal conductance.

CNPase-null mice develop progressive axonal degeneration with (as originally reported; see below) normal-appearing myelin that does not influence axonal action potential propagation (ref. 40 and Figure 2D). Pronounced deficits were described at the paranodal loops and inner tongue processes (40-42). Using novel high-pressure freeze and freeze substitution techniques for electron microscopy, it was found that CNPase is involved in the organization of the actin cytoskeleton surrounding cytoplasmic "myelinic nanochannels" (ref. 19 and Figure 3iii). These myelin channels are noncompacted regions of myelin that allow passage of nutrients, metabolites, and signaling molecules toward the underlying axon $(7,15,19)$. Loss of CNPase severely affected the presence of such cytoplasmic channels, and myelin was far more compacted (ref. 19 and Figure 2D). Interestingly, a decrease in
MBP expression levels (which promotes myelin compaction) rescues the loss of these cytoplasmic channels and rescues the axonal degeneration observed in large-caliber axons of the CNPase-null mice (but not small-caliber axons, where such channels are very rare), indicating that CNPase and MBP have an antagonistic relationship during myelin compaction (19).

In conclusion, oligodendrocyte protein-knockout mouse models suggest that oligodendrocyte myelin is not merely static but far more complex and dynamic than originally thought. These models reveal differential effects of myelin in action potential propagation and axonal degeneration, bringing oligodendrocytes to the forefront of essential players in neuronal metabolic support. The metabolites that are shuttled through the cytoplasmic channels toward the periaxonal space eventually accumulate in the oligodendrocytes if there is no assisted transport to shuttle these metabolites outside the cell. In fact, oligodendrocytes express a specific transporter for glucose-derived metabolites that ensures shuttling of these metabolites toward neurons to meet their metabolic needs (43).

Oligodendrocytes provide neuronal metabolic support through monocarboxylate transporters. Half a decade ago, two seminal publications shed more light on how oligodendrocytes provide metabolic support to neurons $(43,44)$. Firstly, oligodendrocytes were found to express a transporter for monocarboxylate metabolites, monocarboxylate transporter 1 (MCT1), which was previously found to be highly expressed by astrocytes (43). MCTs comprise a superfamily of 16 members of which four members, MCT1, MCT2, MCT3, and MCT4, are expressed in the CNS (reviewed in refs. 45, 46). MCT1-MCT4 cotransport monocarboxylates like lactate, pyruvate, and ketone bodies with $\mathrm{H}^{+}$ions across plasma membranes according to their concentration gradient. This transport ensures delivery of metabolic substrates such as lactate to target cells, where they fuel neuronal energy demands. Different MCTs are differentially expressed in the CNS and have different substrate transport kinetics. MCT1 (with highest affinity for lactate) is prominently expressed in astrocytes and endothelial cells (47-49), whereas MCT2 (with intermediate affinity for lactate) is expressed in neurons $(47,50)$. MCT4 (which has the lowest affinity for lactate) is expressed in astrocytes (50). MCT3 is only expressed at the basal membrane of retinal pigment epithe- 

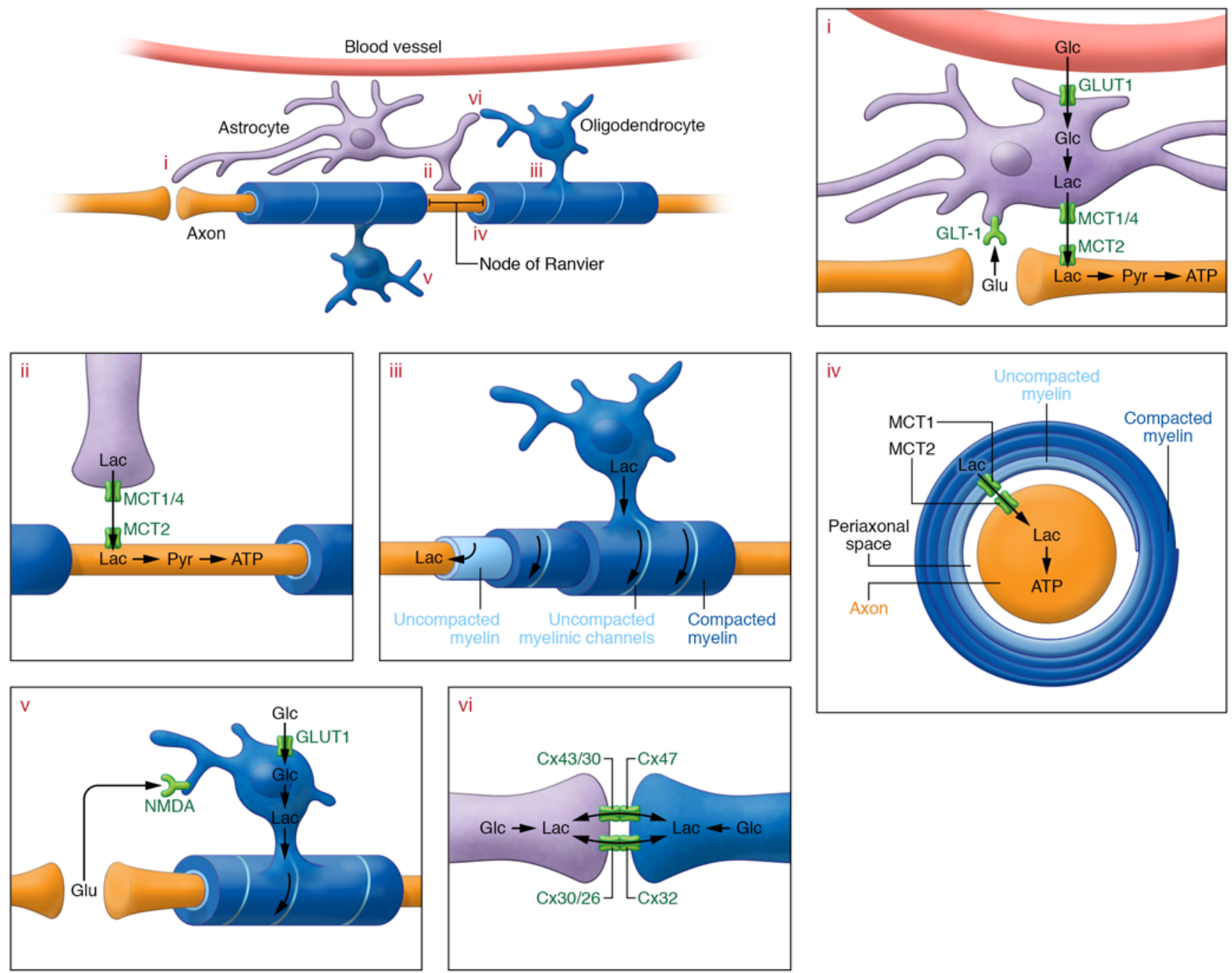

Figure 3. Glial cells provide metabolic support to neurons. (i) At the synapse, neuronal activity releases glutamate (Glu) in the extracellular space, which is taken up by astrocytic GLT-1. Glutamate uptake drives glucose (GIc) uptake through glucose transporters (GLUT1) in astrocytes; glucose is subsequently converted to pyruvate (Pyr) and lactate (Lac). Intracellular lactate can be shuttled to neurons through astrocyte MCT1 and MCT4 and neuronal MCT2. Neuronal lactate can fuel neuronal ATP synthesis in the mitochondria after conversion to pyruvate. (ii) Astrocytes contact neurons at the nodes of Ranvier, and can shuttle metabolic substrates through astrocytic MCT1 and MCT4. Neurons can take up these substrates through MCT2, using them to fuel mitochondrial ATP synthesis. (iii) Oligodendrocyte myelin consists of compacted and uncompacted regions. The latter allow for transport of metabolites within the myelin, toward the adaxonal (nearest to the axon) myelin inner tongue, from where metabolites can be transported to neurons. (iv) Transport occurs through MCT1 expressed at the adaxonal oligodendrocyte process and moves substrates into the periaxonal space, where metabolites can be taken up by neurons through MCT2 and processed for ATP synthesis. (v) The metabolic supportive function of oligodendrocytes is regulated by glutamate binding to the NMDA receptor, followed by enhanced oligodendrocyte glucose uptake and conversion of glucose to lactate, which can then be provided to the neurons. (vi) Another potential source of oligodendrocyte lactate is through gap junction coupling with astrocytes. Whether metabolic supply through this pathway is essential for neuronal metabolic support remains to be determined.

lial cells (51). Early publications suggested that astrocytes play the foremost role in providing neurons with lactate through MCT1 and MCT4 (32, 52); however, it is now well established that MCT1 is strongly expressed by oligodendrocytes at the abaxonal and adaxonal myelinic channels and that oligodendrocyte MCT1 mediates metabolic support to neurons $(43,53)$. Oligodendrocyte-specific MCT1 inhibition in organotypic spinal cord slice cultures leads to axonal injury (Figure 2E and Figure 3iv), which can be rescued by the addition of L-lactate to the culture medium (43). Further, Mct1 heterozygous knockout mice develop axonal injury in the absence of oligodendrocyte injury starting at 7 months of age (43). Lastly, oligodendrocyte-specific MCT1 depletion caused severe axonal injury and motor neuron death in vivo (43). These studies indicate that oligodendrocytes are key mediators of neuronal metabolic support. As oligodendrocytes accumulate intracellular lactate, lactate can flow through MCT1 into the periaxonal space, where neurons can take it up through MCT2 and metabolize lactate to meet their metabolic demands $(43,44,54)$. Other lines of evidence support the idea that lactate flows from oligodendrocytes to neurons. Oligodendrocyte-specific knockout of the mitochondrial respiration protein COX-10, which prevents oxidative metabolism, did not alter myelination, and lactate that had accumulated due to 
ongoing glycolysis was rapidly metabolized within the white matter tracts (44). The study's authors suggested that adult oligodendrocytes can maintain ATP homeostasis with glycolysis alone and that accumulated lactate in oligodendrocytes can either be used to support myelin lipid synthesis in the oligodendrocyte or serve as a lactate source to neurons (44).

It remains to be elucidated how oligodendrocyte MCT1 expression and function are involved in animal models characterized by oligodendrocyte dysfunction (e.g., PLP-null, CNPasenull, and MBP-null mice) or in myelin injury conditions such as multiple sclerosis (MS). Although it does not necessarily affect MCT1 expression, loss of CNPase expression leads to a loss of the noncompacted myelinic channels, which dramatically affect the flow of nutrients reaching the axon. Even if MCT1 expression is not directly altered in these models, MCT1-mediated transfer of lactate to neurons would be dramatically impaired if the normal oligodendrocyte network of myelinic channels or its structural contact with the neuron is no longer maintained.

Lactate is essential for neuronal ATP homeostasis. Whether provided by astrocytes and/or oligodendrocytes, the concept that lactate accumulates in the brain upon increased activation and its importance as a neuronal energy metabolite have been well established $(32,52,55-58)$. The importance of lactate as a metabolic nutrient in vivo was elegantly shown in a study in which rats were subjected to the inhibitory avoidance test (59). Long-term memory formation in the hippocampus of these rats was severely impaired upon hippocampal-specific inhibition of either MCT1, MCT2, or MCT4 expression; MCT1 or MCT4 deficiency could be rescued with injection of L-lactate but not equicaloric glucose. MCT2 inhibition was not rescued by the addition of L-lactate, which is in line with a flow of lactate from glial cell to neuron, where lactate is taken up by neuron-specific MCT2 and metabolized (59). The cellular source of the lactate that provides long-term memory benefits was originally suggested to be astrocytes; however, the subsequent identification of MCT1 expression in oligodendrocytes suggests that oligodendroglia also may mediate these effects (43). Lactate has been shown to have neuroprotective effects in brain injury models, either as a neuronal metabolite or as a signaling molecule that binds the neuronal lactate receptor HCA1 (GPR81) (60-64). This finding underscores the potential role of lactate in models of neurodegeneration wherein neurons are metabolically stressed. More recent studies have demonstrated dynamic changes in lactate flow. Using live in vivo imaging of a lactate fluorescence resonance energy transfer (FRET) reporter construct, intravenously injected lactate was found to accumulate more in neurons than in astrocytes (65). Interestingly, neuronal lactate levels remained unchanged upon peripheral lactate injection, which was expected to cause intracellular lactate depletion through trans-acceleration (65). These data suggest an actual flow of lactate from astrocyte to neurons, as postulated by the astrocyte-neuron lactate shuttle. Unfortunately, this study did not analyze other glial cells types, such as oligodendrocytes, which could provide neurons with metabolic support.

Other studies in humans and mouse models have suggested that neurons metabolize lactate and might prefer lactate over glucose to fuel energy metabolism (66-68). This is further supported by a study in which neuronal ATP generation was measured in acutely isolated optic nerves using a fluorescent ATP reporter dye (69). The electrical activity of the neurons was highly dependent on lactate metabolism even in the presence of glucose, as the drop in ATP levels observed upon high-frequency stimulation was significantly higher upon inhibition of lactate metabolism either using lactate transport inhibitors or by addition of the nonphysiological D-lactate to the extracellular environment (69).

Role of lactate in myelination. As MCT1-mediated transport is bidirectional, lactate has also been suggested to be a potential source for oligodendrocyte ATP homeostasis important for the generation and maintenance of myelin integrity, a huge metabolic burden on the oligodendrocyte energy levels $(70,71)$. One study has shown that lactate added to rat cerebellar or cortical slices is highly metabolized by MCT1-expressing oligodendrocytes. When these slices were grown in low-glucose conditions, lactate is highly metabolized by MCT1-expressing oligodendrocytes in the cerebellum and corpus callosum (70). In cultured cortical slices grown in low-glucose conditions, it was found that myelination and oligodendrogenesis were severely impaired, but rescued by lactate supplementation, suggesting that lactate is important for oligodendrocyte maturation and myelination (70). However, the in vivo relevance of these findings and the need for lactate to promote myelination or oligodendrogenesis is uncertain. In $\mathrm{Mct}^{+/-}$mice, oligodendrogenesis and myelination are completely normal (Figure 2E), arguing against a role for MCT1 in myelin homeostasis either during development or in adulthood (43). It remains to be elucidated whether homozygous knockout of Mct1 in oligodendrocyte-lineage cells impairs developmental myelination and/or myelin maintenance in vivo. Alternatively, lactate and other metabolites could bypass MCT1 and reach oligodendrocytes through either homotypic or heterotypic gap junctions (see below). In addition, oligodendrocytes express glucose transporter 1 (GLUT1), through which glucose can enter the cell and contribute to lipid synthesis in oligodendrocytes either as a precursor or by generating ATP during glycolysis $(44,72)$.

NMDA receptor signaling regulates oligodendrocyte-mediated metabolic support. The actual source of the lactate accumulating in the oligodendrocytes is still under debate. Oligodendrocytes could take up glucose using the cell surface-expressed GLUT1 and then convert glucose into lactate through glycolysis. A recent elegant study supports a mechanism in which NMDA receptors (NMDARs) are involved in oligodendrocyte-mediated neuronal metabolic support (ref. 72 and Figure 3v). Oligodendrocyte-lineage cells express NMDARs, but their importance has remained enigmatic, as deletion of specific NMDAR subtypes had no influence on OPC proliferation, myelination, the ability of OPCs to form synapses with glutamatergic neurons, or their susceptibility to experimental autoimmune encephalomyelitis $(73,74)$. NMDARs are expressed on the noncompacted cell surface of oligodendrocytes and respond to neuronal activity with enhanced $\mathrm{Ca}^{2+}$ influx and increased glucose uptake through GLUT1 (72). When ex vivo preparations of acutely isolated optic nerves of NMDA-mutant mice were exposed to glucose and oxygen deprivation, there was reduced functional recovery when they were returned to glucose and oxygen exposure as compared with wild-type optic nerves. Functional recovery was observed upon exposure to lactate and oxygen, a condition that would bypass the need for oligodendrocyte-derived lactate to provide metabolic support to neurons. 
These data suggest that oligodendrocyte metabolic support is partly mediated by activity-dependent glutamate release from neurons, binding of glutamate to oligodendrocyte NMDARs, and increased glucose uptake and lactate production in oligodendrocytes. These data strongly indicate that the oligodendrocyteneuron lactate shuttle is driven by neuronal activity, which controls the production of lactate at concentrations needed to address neuronal energy demands.

Connexin gap junction coupling in oligodendrocyte metabolic support. As an alternative to cell surface glucose transporters, oligodendrocytes can be provided with metabolic nutrients through gap junction coupling with other cells like astrocytes (refs. 75, 76; reviewed in refs. 54, 77, 78; and Figure 3vi). Astrocytes are well connected to the vasculature through their membranous end-feet and respond to increased metabolic demand with enhanced glucose uptake. Apart from their ability to shuttle glucose-derived metabolites to neurons as predicted by the astrocyte-neuron lactate shuttle hypothesis (see above), astrocyte-derived lactate and glucose could shuttle into oligodendrocytes through gap junctions composed of connexin (Cx) hemichannels. Astrocytes express Cx43, Cx26, and Cx30 hemichannels, which form gap junctions with other astrocyte hemichannels, as well as with neuronal (Cx36) and oligodendroglial hemichannels (Cx32 and Cx47) (79). Oligodendrocytes connect with astrocytes through four pairs of heterotypic gap junctions: $\mathrm{Cx} 47-\mathrm{Cx} 43, \mathrm{Cx} 47-\mathrm{Cx} 30, \mathrm{Cx} 32-\mathrm{Cx} 30$, and Cx32-Cx26 (80). The true importance of the metabolic crosstalk between astrocytes and oligodendrocytes is only beginning to be understood. Mouse models lacking oligodendrocyte $\mathrm{Cx} 47$ show very mild changes in the myelin (despite $80 \%$ loss of cell coupling and total loss of astrocyte-to-oligodendrocyte coupling; ref. 81), whereas Cx32-knockout mice do not develop a CNS phenotype at all, but do develop a severe peripheral nervous system demyelination $(82,83)$. In contrast, double oligodendrocyte $C \times 47 / C \times 32$ knockouts develop a very severe dysmyelinating phenotype with early lethality $(82,84)$, indicating that oligodendrocyte connexins are essential for proper myelination. Similarly, loss of astrocyte connexin expression affects oligodendrocytes as well. Targeted loss of astrocyte $\mathrm{Cx} 43$ expression inhibits glucose delivery to OPCs and OPC proliferation, which in turn can influence oligodendrogenesis and oligodendrocyte metabolic support (76). In addition, double knockouts for the astrocyte hemichannels Cx43 and Cx30 develop widespread white matter pathology with impairment of oligodendrocyte maturation and myelin vacuolation, whereas mice with astrocyte-targeted loss of $\mathrm{Cx} 43$ or $\mathrm{Cx} 30$-null mice do not show signs of abnormal myelination (85-87). Double knockouts affecting both oligodendrocyte and astrocyte hemichannels (Cx47) Cx30) develop a progressive phenotype with early vacuolization of myelin with microgliosis, astrogliosis, motor impairment, and early death (88). Glial fibrillary acidic protein (Gfap)-Cre-mediated depletion of astrocyte $\mathrm{Cx} 43$ in oligodendrocyte Cx32-null mice revealed a similar phenotype characterized by severe myelin vacuolization, astrocyte loss, and early death (89).

The findings described above indicate that glial networks involving astrocyte-to-oligodendrocyte coupling are essential for normal myelination. Whether a metabolic disconnection between oligodendrocytes and astrocytes in these models contributes to the phenotype observed remains to be established; in fact, many other potential contributors such as changes in extracellular $\mathrm{K}^{+}$concentration due to altered $\mathrm{K}^{+}$buffering or changes in propagation of $\mathrm{Ca}^{2+}$ waves could contribute as well $(90,91)$. The finding that loss of connexin coupling between astrocytes and oligodendrocytes mostly affects early myelination is in correlation with glucose/lactate entering the oligodendrocytes and being used by oligodendrocytes either to serve their own metabolic demands or to serve as a precursor for fatty acid and lipid synthesis during myelination (70). After myelination is completed, continued glycolysis in oligodendrocytes and accumulation of lactate (through oligodendrocyte glycolysis in response to NMDAR signaling or through import through astrocyte-oligodendrocyte gap junctions) could serve as a reservoir of metabolites that neurons can use to support their metabolic needs $(44,72)$. Studies targeting astrocyte-oligodendrocyte coupling after completion of myelination could shed light on whether these astrocyte-oligodendrocyte gap junctions are truly essential to maintain oligodendrocyte metabolic support.

\section{Involvement of oligodendrocyte metabolic support in neurodegenerative diseases}

Multiple sclerosis. Oligodendrocytes are well known to be a primary target in certain neurodegenerative diseases, such as MS. MS is a chronic neuroinflammatory disease that is characterized by profound inflammation, damage to oligodendrocytes with ensuing demyelination, and death of axons (reviewed in refs. 92, 93). These pathologies lead to severe symptoms in patients ranging from neuropsychological problems and cognitive decline to weakness, altered motor coordination issues, and sensory disturbances - all reflecting damage to axonal connections between brain and spinal cord regions. Demyelination is concomitant with remyelination, with the extent of remyelination variable among MS patients as found upon postmortem examination of white matter regions (94). As oligodendrocytes in MS become impaired and axons lose a functional compacted myelin sheath, one would predict that oligodendrocyte metabolic support would fail. Interestingly, in the early stages of a mouse model of MS, experimental autoimmune encephalomyelitis (EAE), there is evidence that axonal damage can present itself well before these axons become demyelinated, suggesting that loss of oligodendrocyte metabolic support is a very early event in the pathogenesis (95). Changes in MCT expression have been observed in MS patients. MCT1 expression seems to be upregulated in active MS lesions in astrocytes, whereas neuronal MCT2 is markedly downregulated, which could profoundly impede metabolic support to neurons (96). In addition, changes in astrocyte/oligodendrocyte connexins have been reported in MS patients and in MS animal models (97-99): In MS patients, Cx32 and Cx47 gap junctions are downregulated, while $\mathrm{Cx} 43$ expression is upregulated. This finding is not surprising given that oligodendrocytes are damaged in MS plaques, whereas astrocytes become more reactive. In the EAE mouse model, connexin expression is quite dynamic: $\mathrm{Cx} 43, \mathrm{Cx} 47$, and Cx32 were severely reduced within demyelinated lesions, but increased in remyelinating regions (98); similar observations were made in the cuprizone mouse model (100). As discussed earlier, loss of astrocyte $\mathrm{Cx} 43 / \mathrm{Cx} 30$ suppresses oligodendrocyte maturation, and loss of astrocyte $\mathrm{Cx} 43$ inhibits OPC proliferation $(76,85)$. These data suggest that in MS and other diseases in 
which oligodendrocytes are involved, glial networking through connexin hemichannels could play an important role in the demyelination/remyelination observed in MS patients. Therapeutic approaches in MS that promote maturation of OPCs into mature oligodendrocytes or improve oligodendrocyte survival could generate oligodendrocytes that provide trophic support to neurons, for example, by improving the oligodendrocyte-neuron interaction, even without directly regulating the mechanisms (described in previous sections) that affect oligodendrocyte metabolic support. In fact, altering the activity of many different signaling pathways involved in remyelination, including Lingo-1, Wnt signaling, and acetylcholine receptor antagonists, or prolonging the cellular stress response, has been shown to enhance remyelination and improve symptoms in MS mouse models (reviewed in ref. 101). Some of these therapeutic studies performed in mice have led to the initiation of clinical trials in MS patients: A humanized antibody therapy targeting Lingo-1 (BIBO33) has been tested in a phase II clinical trial with inconclusive results (ClinicalTrials.gov, NCT01864148). Therapeutic trials with guanabenz (which stabilizes the cellular stress response; NCT02423083) and clemastine (an anticholinergic drug; NCT02040298 and NCT02521311) are currently ongoing. It remains to be investigated how the drugs that enhance remyelination affect the mechanisms involved in oligodendrocyte metabolic support. Recently miR-219 was shown to enhance oligodendrocyte MCT1 expression and promote oligodendrogenesis and myelination in the cuprizone mouse model (102). MCT1 was suggested to be mediating the enhanced remyelination, but as the authors used a nonspecific MCT1 inhibitor in this study, the true importance of MCT1 in remyelination remains enigmatic. New tools that allow for modulating the function of the different mechanisms involved in oligodendrocyte metabolic support will shed more light on its importance in mouse models of demyelination.

Amyotrophic lateral sclerosis. Amyotrophic lateral sclerosis (ALS) is a degenerative motor neuron disease characterized by the death of motor neurons in the motor cortex, brain stem, and spinal cord, leading to progressive paralysis and death, generally within 3-5 years after disease onset (reviewed in refs. 103, 104). No cure or treatment is currently available, although two drugs (riluzole and edaravone) increase survival and slightly slow disease $(105,106)$. Although ALS is classically defined as a motor neuron disease, it is now well established that other neuronal cell populations are at risk in certain CNS areas, as up to $50 \%$ of patients develop a mild dementia and some genetic forms of the disease (e.g., C9ORF72 mutations) are associated with frontotemporal dementia. Notably, oligodendrocytes also are severely affected. Luxol fast blue staining of postmortem tissue from sporadic ALS patients indicates the presence of large patches of demyelinated CNS tissue (107), and protein expression studies indicate the loss of several oligodendrocyte protein markers, including MBP and CNPase (107). The impact of oligodendrocyte degeneration on motor neuron death has recently been elucidated. In mouse models of ALS, overexpressing a disease-causing SOD1 ${ }^{\mathrm{G} 93 \mathrm{~A}}$ mutant triggered oligodendrocyte degeneration and death; these cells were not replaced by newly generated functional oligodendrocytes $(107,108)$. Interestingly, deletion of the disease-causing SOD1 ${ }^{\mathrm{G} 93 \mathrm{~A}}$ mutant from OPCs and oligodendrocytes significantly delayed disease onset and extended survival, representing one of the strongest disease effects published to date (107). These findings indicate that OPCs and oligodendrocytes critically alter motor neuron viability and survival in this model. A later study indicated that human induced pluripotent stem cell-derived (iPSC-derived) oligodendrocytes contribute to the loss of motor neurons in vitro (109). Validating the prior in vivo studies, motor neuron loss was characterized by a decrease in oligodendrocyte lactate release and MCT1 expression. Interestingly, motor neuron loss was rescued by SOD1 knockdown in both familial and sporadic ALS OPCs (not in C9ORF72 mutant carriers), but not by knockdown in mature oligodendrocytes. This study underscores the importance of oligodendrocyte-lineage cells, especially OPCs, in causing motor neuron toxicity in mutant SOD1 and sporadic ALS. As oligodendrocytes degenerate and die, MCT1 expression is lost, which disrupts oligodendrocyte-mediated metabolic support of neurons. This loss of metabolic support could accelerate the demise of the motor neurons and markedly affect survival.

Changes in oligodendrocyte and astrocyte connexin expression could affect oligodendrocyte and neuronal metabolic support in ALS patients and disease models. In mutant SOD1 animals, astrocyte $\mathrm{Cx} 43$ is significantly upregulated with disease progression, whereas oligodendrocyte $\mathrm{Cx} 32$ and $\mathrm{Cx} 47$ are downregulated $(110,111)$. These changes in glial network activity through connexin hemichannels could contribute to the failure of remyelination and eventual demise of motor neurons. More studies using either knockout or overexpression models for MCT1 and astrocyte/oligodendrocyte connexins could elucidate the importance of these pathways in the pathogenesis of motor neuron disease.

\section{Therapeutic potential}

Oligodendrocyte degeneration and death as observed in MS and ALS and mouse models of these diseases would not only impair axonal conductance but also metabolic support to the at-risk neurons. Therapies that have already shown efficacy in promoting remyelination could be very efficient in restoring oligodendrocyte metabolic support simply by improvement of the connectivity between oligodendrocytes and neurons. Another strategy to overcome the loss of oligodendrocyte metabolic support is to upregulate MCT1 expression or by increasing the flow of metabolic nutrients through the noncompacted myelin nanochannels. Alternatively, one could try to enhance the shuttling of metabolites through connexin gap junctions, or enhance NMDA signaling in oligodendrocytes to increase glucose uptake through oligodendrocyte GLUT1. Such approaches would be useful only in contexts where the normal flow of metabolites toward the periaxonal space is not interrupted; for example, MCT1 upregulation would not show any benefit to axons that have entirely lost their insulating myelin sheath, but would only be useful when the normal oligodendrocyte-to-neuron connectivity is more or less maintained, such as early in disease pathogenesis. Alternatively, promoting specific changes in the expression of the genes involved in oligodendrocyte axonal support could also affect oligodendrocyte energy homeostasis and in turn promote the maintenance of the myelin sheath, which would therefore not impede the flow of trophic support from oligodendrocyte to neuron. Whether the noncompacted myelin channels are affected in certain neurodegenerative diseases like MS and ALS, and how demyelination affects 
the presence and functioning of these channels, remain to be elucidated. Similarly, whether oligodendrocyte NMDAR signaling is altered in these diseases requires further investigation, though one study in the cuprizone mouse model of demyelination did not find any changes in the presentation of the phenotype upon depletion of NMDAR subunits in oligodendrocyte lineage cells (73). New in vivo tools using, for instance, conditional deletion or overexpression of the pathways involved in oligodendrocyte metabolic support could shed light on its importance in models characterized by demyelination and/or axonal degeneration.

\section{Concluding remarks}

For several decades, it has been well established that neurons need additional metabolic support to sustain their normal homeostasis. Astrocytes provide neuronal support by transferring glycolytic derivatives of glucose, pyruvate, and lactate, and shuttle these nutrients to neurons through MCTs. More recently, it has been shown that oligodendrocytes have a very similar role in supplying neurons with nutrients: oligodendrocyte-specific knockdown of myelin proteins and MCT1 has clearly shown that oligodendrocytes provide metabolic support to neurons. Although compact myelin could prevent the transfer of such nutrients throughout the oligodendrocyte cytoplasm, oligodendrocytes were found to allow such transfer through local regions of uncompacted myelin. In certain neurodegenerative diseases, this metabolic supportive function could become compromised and contribute to the degeneration and death of certain neuronal subpopulations. More research is needed to understand the role of oligodendrocyte metabolic support, including transfer of metabolites through myelinic channels, MCT1, and connexin hemichannel connections to other oligodendrocytes and astrocytes. Such studies will lead us to better appreciate the importance of oligodendrocytes in neuronal homeostasis, neuronal degeneration, and potential therapeutic strategies.

\section{Acknowledgments}

This work was supported by grants from the Muscular Dystrophy Association, the NIH (grants R01NS099320 and R01NS09423), Target ALS, the ALS Association, and the Robert Packard Center for ALS Research.

Address correspondence to: Jeffrey D. Rothstein, The John G. Rangos Sr. Building, 855 North Wolfe Street, Room 270, 2nd Floor, Baltimore, Maryland 21205, USA. Phone: 410.614.3846; Email: jrothstein@jhmi.edu.
1. Kessaris N, Fogarty M, Iannarelli P, Grist M, Wegner M, Richardson WD. Competing waves of oligodendrocytes in the forebrain and postnatal elimination of an embryonic lineage. Nat Neurosci. 2006;9(2):173-179.

2. Pringle NP, Richardson WD. A singularity of PDGF alpha-receptor expression in the dorsoventral axis of the neural tube may define the origin of the oligodendrocyte lineage. Development. 1993;117(2):525-533.

3. Bergles DE, Richardson WD. Oligodendrocyte development and plasticity. Cold Spring Harb Perspect Biol. 2015;8(2):a020453.

4. Rivers LE, et al. PDGFRA/NG2 glia generate myelinating oligodendrocytes and piriform projection neurons in adult mice. Nat Neurosci. 2008;11(12):1392-1401.

5. Psachoulia K, Jamen F, Young KM, Richardson WD. Cell cycle dynamics of NG2 cells in the postnatal and ageing brain. Neuron Glia Biol. 2009;5(3-4):57-67.

6. Chang KJ, Redmond SA, Chan JR. Remodeling myelination: implications for mechanisms of neural plasticity. Nat Neurosci. 2016;19(2):190-197.

7. Simons M, Nave KA. Oligodendrocytes: myelination and axonal support. Cold Spring Harb Perspect Biol. 2015;8(1):a020479.

8. Snaidero N, Simons M. The logistics of myelin biogenesis in the central nervous system. Glia. 2017;65(7):1021-1031.

9. Young KM, et al. Oligodendrocyte dynamics in the healthy adult CNS: evidence for myelin remodeling. Neuron. 2013;77(5):873-885.

10. Schneider S, et al. Decrease in newly generated oligodendrocytes leads to motor dysfunctions and changed myelin structures that can be rescued by transplanted cells. Glia. 2016;64(12):2201-2218.

11. Kang SH, Fukaya M, Yang JK, Rothstein JD, Bergles DE. NG2+ CNS glial progenitors remain committed to the oligodendrocyte lineage in postnatal life and following neurodegeneration. Neuron. 2010;68(4):668-681.

12. Tomassy GS, et al. Distinct profiles of myelin distribution along single axons of pyramidal neurons in the neocortex. Science. 2014;344(6181):319-324.

13. Gaesser JM, Fyffe-Maricich SL. Intracellular signaling pathway regulation of myelination and remyelination in the CNS. Exp Neurol. 2016;283(pt B):501-511.

14. Emery B, Lu QR. Transcriptional and epigenetic regulation of oligodendrocyte development and myelination in the central nervous system. Cold Spring Harb Perspect Biol. 2015;7(9):a020461.

15. Snaidero N, et al. Myelin membrane wrapping of CNS axons by $\mathrm{PI}(3,4,5) \mathrm{P} 3$-dependent polarized growth at the inner tongue. Cell. 2014;156(1-2):277-290.

16. Zuchero JB, et al. CNS myelin wrapping is driven by actin disassembly. Dev Cell. 2015;34(2):152-167.

17. Nawaz S, et al. Actin filament turnover drives leading edge growth during myelin sheath formation in the central nervous system. Dev Cell. 2015;34(2):139-151.

18. Aggarwal S, et al. A size barrier limits protein diffusion at the cell surface to generate lipid-rich myelinmembrane sheets. Dev Cell. 2011;21(3):445-456.

19. Snaidero N, et al. Antagonistic functions of MBP and CNP establish cytosolic channels in CNS myelin. Cell Rep. 2017;18(2):314-323.

20. Xiao L, et al. Rapid production of new oligodendrocytes is required in the earliest stages of motor-skill learning. Nat Neurosci. 2016;19(9):1210-1217.

21. McKenzie IA, et al. Motor skill learning requires active central myelination. Science. 2014;346(6207):318-322.

22. Gibson EM, et al. Neuronal activity promotes oligodendrogenesis and adaptive myelination in the mammalian brain. Science. 2014;344(6183):1252304

23. Sampaio-Baptista C, et al. Motor skill learning induces changes in white matter microstructure and myelination. J Neurosci 2013;33(50):19499-19503.

24. Fields RD. A new mechanism of nervous system plasticity: activity-dependent myelination. Nat Rev Neurosci. 2015;16(12):756-767.

25. Koudelka S, et al. Individual neuronal subtypes exhibit diversity in CNS myelination mediated by synaptic vesicle release. Curr Biol. 2016;26(11):1447-1455.

26. Scholz J, Klein MC, Behrens TE, Johansen-Berg H. Training induces changes in white-matter architecture. Nat Neurosci. 2009;12(11):1370-1371.

27. Bengtsson SL, Nagy Z, Skare S, Forsman L, Forssberg H, Ullén F. Extensive piano practicing has regionally specific effects on white matter development. Nat Neurosci. 2005;8(9):1148-1150.

28. Almeida A, Almeida J, Bolaños JP, Moncada S. Different responses of astrocytes and neurons to nitric oxide: the role of glycolytically generated ATP in astrocyte protection. Proc Natl Acad Sci US A. 2001;98(26):15294-15299.

29. Saez I, et al. Neurons have an active glycogen metabolism that contributes to tolerance to hypoxia. J Cereb Blood Flow Metab. 2014;34(6):945-955.

30. Young EA, et al. Imaging correlates of decreased axonal $\mathrm{Na}^{+} / \mathrm{K}^{+}$ATPase in chronic multiple sclerosis lesions. Ann Neurol. 2008;63(4):428-435.

31. Ohno N, et al. Myelination and axonal electrical activity modulate the distribution and motility of mitochondria at CNS nodes of Ranvier. J Neurosci. 2011;31(20):7249-7258.

32. Pellerin L, Magistretti PJ. Glutamate uptake into astrocytes stimulates aerobic glycolysis: a mechanism coupling neuronal activity to glucose utilization. Proc Natl Acad Sci U S A. 
1994;91(22):10625-10629.

33. Kasischke KA, Vishwasrao HD, Fisher PJ, Zipfel WR, Webb WW. Neural activity triggers neuronal oxidative metabolism followed by astrocytic glycolysis. Science. 2004;305(5680):99-103.

34. Lerchundi R, et al. $\mathrm{NH} 4(+)$ triggers the release of astrocytic lactate via mitochondrial pyruvate shunting. Proc Natl Acad Sci US A. 2015;112(35):11090-11095.

35. Griffiths I, et al. Axonal swellings and degeneration in mice lacking the major proteolipid of myelin. Science. 1998;280(5369):1610-1613.

36. Klugmann M, et al. Assembly of CNS myelin in the absence of proteolipid protein. Neuron. 1997;18(1):59-70.

37. Loers G, Aboul-Enein F, Bartsch U, Lassmann H, Schachner M. Comparison of myelin, axon, lipid, and immunopathology in the central nervous system of differentially myelin-compromised mutant mice: a morphological and biochemical study. Mol Cell Neurosci. 2004;27(2):175-189.

38. Joshi DC, et al. Deletion of mitochondrial anchoring protects dysmyelinating shiverer: implications for progressive MS. J Neurosci. 2015;35(13):5293-5306.

39. Chernoff GF. Shiverer: an autosomal recessive mutant mouse with myelin deficiency. J Hered. 1981;72(2):128.

40. Lappe-Siefke C, et al. Disruption of Cnp1 uncouples oligodendroglial functions in axonal support and myelination. Nat Genet. 2003;33(3):366-374.

41. Rasband MN, et al. CNP is required for maintenance of axon-glia interactions at nodes of Ranvier in the CNS. Glia. 2005;50(1):86-90.

42. Edgar JM, et al. Early ultrastructural defects of axons and axon-glia junctions in mice lacking expression of Cnp1. Glia. 2009;57(16):1815-1824.

43. Lee $\mathrm{Y}$, et al. Oligodendroglia metabolically support axons and contribute to neurodegeneration. Nature. 2012;487(7408):443-448.

44. Fünfschilling U, et al. Glycolytic oligodendrocytes maintain myelin and long-term axonal integrity. Nature. 2012;485(7399):517-521.

45. Halestrap AP. Monocarboxylic acid transport. Compr Physiol. 2013;3(4):1611-1643.

46. Halestrap AP, Wilson MC. The monocarboxylate transporter family - role and regulation. IUBMB Life. 2012;64(2):109-119.

47. Pierre K, Pellerin L, Debernardi R, Riederer BM, Magistretti PJ. Cell-specific localization of monocarboxylate transporters, MCT1 and MCT2, in the adult mouse brain revealed by double immunohistochemical labeling and confocal microscopy. Neuroscience. 2000;100(3):617-627.

48. Bröer S, et al. Comparison of lactate transport in astroglial cells and monocarboxylate transporter 1 (MCT 1) expressing Xenopus laevis oocytes. Expression of two different monocarboxylate transporters in astroglial cells and neurons. J Biol Chem. 1997;272(48):30096-30102.

49. Gerhart DZ, Enerson BE, Zhdankina OY, Leino RL, Drewes LR. Expression of monocarboxylate transporter MCT1 by brain endothelium and glia in adult and suckling rats. Am JPhysiol. 1997;273(1 pt 1):E207-E213.

50. Rafiki A, Boulland JL, Halestrap AP, Ottersen OP, Bergersen L. Highly differential expression of the monocarboxylate transporters MCT2 and
MCT4 in the developing rat brain. Neuroscience. 2003;122(3):677-688.

51. Philp NJ, Yoon H, Grollman EF. Monocarboxylate transporter MCT1 is located in the apical membrane and MCT3 in the basal membrane of rat RPE. Am JPhysiol. 1998;274(6 pt 2):R1824-R1828.

52. Tekkök SB, Brown AM, Westenbroek R, Pellerin L, Ransom BR. Transfer of glycogen-derived lactate from astrocytes to axons via specific monocarboxylate transporters supports mouse optic nerve activity. J Neurosci Res. 2005;81(5):644-652.

53. Saab AS, Nave KA. Neuroscience: A mechanism for myelin injury. Nature. 2016;529(7587):474-475.

54. Morrison BM, Lee Y, Rothstein JD. Oligodendroglia: metabolic supporters of axons. Trends Cell Biol. 2013;23(12):644-651.

55. Hu Y, Wilson GS. A temporary local energy pool coupled to neuronal activity: fluctuations of extracellular lactate levels in rat brain monitored with rapid-response enzyme-based sensor. J Neurochem. 1997;69(4):1484-1490.

56. Pellerin L, Magistretti PJ. Sweet sixteen for ANLS JCereb Blood Flow Metab. 2012;32(7):1152-1166.

57. Brown AM, Wender R, Ransom BR. Metabolic substrates other than glucose support axon function in central white matter. JNeurosci Res. 2001;66(5):839-843.

58. Wender R, Brown AM, Fern R, Swanson RA, Farrell K, Ransom BR. Astrocytic glycogen influences axon function and survival during glucose deprivation in central white matter. J Neurosci. 2000;20(18):6804-6810.

59. Suzuki A, et al. Astrocyte-neuron lactate transport is required for long-term memory formation. Cell. 2011;144(5):810-823.

60. Schurr A. Lactate, glucose and energy metabolism in the ischemic brain (Review). Int JMol Med. 2002;10(2):131-136.

61. Castillo X, et al. A probable dual mode of action for both L- and D-lactate neuroprotection in cerebral ischemia. JCereb Blood Flow Metab. 2015;35(10):1561-1569.

62. Schurr A, Payne RS, Miller JJ, Tseng MT. Preischemic hyperglycemia-aggravated damage: evidence that lactate utilization is beneficial and glucose-induced corticosterone release is detrimental. J Neurosci Res. 2001;66(5):782-789.

63. Mosienko V, Teschemacher AG, Kasparov S. Is L-lactate a novel signaling molecule in the brain? JCereb Blood Flow Metab. 2015;35(7):1069-1075.

64. Bergersen LH. Lactate transport and signaling in the brain: potential therapeutic targets and roles in body-brain interaction. J Cereb Blood Flow Metab. 2015;35(2):176-185.

65. Mächler P, et al. In vivo evidence for a lactate gradient from astrocytes to neurons. Cell Metab. 2016;23(1):94-102.

66. Wyss MT, Jolivet R, Buck A, Magistretti PJ, Weber B. In vivo evidence for lactate as a neuronal energy source. J Neurosci. 2011;31(20):7477-7485.

67. Boumezbeur F, et al. The contribution of blood lactate to brain energy metabolism in humans measured by dynamic $13 \mathrm{C}$ nuclear magnetic resonance spectroscopy. J Neurosci. 2010;30(42):13983-13991.

68. Glenn TC, et al. Lactate: brain fuel in human traumatic brain injury: a comparison with normal healthy control subjects. JNeurotrauma.
2015;32(11):820-832.

69. Trevisiol A, et al. Monitoring ATP dynamics in electrically active white matter tracts. Elife. 2017;6:e24241.

70. Rinholm JE, Hamilton NB, Kessaris N, Richardson WD, Bergersen LH, Attwell D. Regulation of oligodendrocyte development and myelination by glucose and lactate. J Neurosci. 2011;31(2):538-548.

71. Harris JJ, Attwell D. The energetics of CNS white matter. J Neurosci. 2012;32(1):356-371.

72. Saab AS, et al. Oligodendroglial NMDA receptors regulate glucose import and axonal energy metabolism. Neuron. 2016;91(1):119-132.

73. Guo F, et al. Disruption of NMDA receptors in oligodendroglial lineage cells does not alter their susceptibility to experimental autoimmune encephalomyelitis or their normal development. J Neurosci. 2012;32(2):639-645.

74. De Biase LM, et al. NMDA receptor signaling in oligodendrocyte progenitors is not required for oligodendrogenesis and myelination. J Neurosci. 2011;31(35):12650-12662.

75. Rouach N, Koulakoff A, Abudara V, Willecke K, Giaume C. Astroglial metabolic networks sustain hippocampal synaptic transmission. Science. 2008;322(5907):1551-1555.

76. Niu J, et al. Connexin-based channels contribute to metabolic pathways in the oligodendroglial lineage. JCell Sci. 2016;129(9):1902-1914.

77. Li T, Giaume C, Xiao L. Connexins-mediated glia networking impacts myelination and remyelination in the central nervous system. Mol Neurobiol. 2014;49(3):1460-1471.

78. Hirrlinger J, Nave KA. Adapting brain metabolism to myelination and long-range signal transduction. Glia. 2014;62(11):1749-1761.

79. Nagy JI, Dudek FE, Rash JE. Update on connexins and gap junctions in neurons and glia in the mammalian nervous system. Brain Res Brain Res Rev. 2004;47(1-3):191-215.

80. Magnotti LM, Goodenough DA, Paul DL. Functional heterotypic interactions between astrocyte and oligodendrocyte connexins. Glia. 2011;59(1):26-34

81. Maglione M, et al. Oligodendrocytes in mouse corpus callosum are coupled via gap junction channels formed by connexin 47 and connexin 32 . Glia. 2010;58(9):1104-1117.

82. Odermatt B, et al. Connexin 47 (Cx47)-deficient mice with enhanced green fluorescent protein reporter gene reveal predominant oligodendrocytic expression of $\mathrm{Cx} 47$ and display vacuolized myelin in the CNS. J Neurosci. 2003;23(11):4549-4559.

83. Sargiannidou I, et al. Connexin 32 mutations cause loss of function in Schwann cells and oligodendrocytes leading to PNS and CNS myelination defects. J Neurosci. 2009;29(15):4736-4749.

84. Menichella DM, Goodenough DA, Sirkowski E, Scherer SS, Paul DL. Connexins are critical for normal myelination in the CNS. JNeurosci. 2003;23(13):5963-5973.

85. Lutz SE, Zhao Y, Gulinello M, Lee SC, Raine CS, Brosnan CF. Deletion of astrocyte connexins 43 and 30 leads to a dysmyelinating phenotype and hippocampal CA1 vacuolation. J Neurosci. 2009;29(24):7743-7752.

86. Nakase T, Söhl G, Theis M, Willecke K, Naus CC. 
Increased apoptosis and inflammation after focal brain ischemia in mice lacking connexin 43 in astrocytes. Am J Pathol. 2004;164(6):2067-2075.

87. Dere E, et al. Connexin30-deficient mice show increased emotionality and decreased rearing activity in the open-field along with neurochemical changes. Eur J Neurosci. 2003;18(3):629-638.

88. Tress O, et al. Panglial gap junctional communication is essential for maintenance of myelin in the CNS. J Neurosci. 2012;32(22):7499-7518.

89. Magnotti LM, Goodenough DA, Paul DL. Deletion of oligodendrocyte $\mathrm{Cx} 32$ and astrocyte $\mathrm{Cx} 43$ causes white matter vacuolation, astrocyte loss and early mortality. Glia. 2011;59(7):1064-1074.

90. Parys B, Côté A, Gallo V, De Koninck P, Sík A. Intercellular calcium signaling between astrocytes and oligodendrocytes via gap junctions in culture. Neuroscience. 2010;167(4):1032-1043.

91. Menichella DM, et al. Genetic and physiological evidence that oligodendrocyte gap junctions contribute to spatial buffering of potassium released during neuronal activity. J Neurosci. 2006;26(43):10984-10991.

92. Dendrou CA, Fugger L, Friese MA. Immunopathology of multiple sclerosis. Nat Rev Immunol. 2015;15(9):545-558.

93. Mahad DH, Trapp BD, Lassmann H. Pathological mechanisms in progressive multiple sclerosis. Lancet Neurol. 2015;14(2):183-193.

94. Patrikios P, et al. Remyelination is extensive in a subset of multiple sclerosis patients. Brain. 2006;129(pt 12):3165-3172.

95. Nikić I, et al. A reversible form of axon damage in experimental autoimmune encephalomyelitis and multiple sclerosis. Nat Med. 2011;17(4):495-499.

96. Nijland PG, et al. Cellular distribution of glucose and monocarboxylate transporters in human brain white matter and multiple sclerosis lesions. Glia. 2014;62(7):1125-1141.

97. Masaki K. Early disruption of glial communication via connexin gap junction in multiple sclerosis, Baló's disease and neuromyelitis optica. Neuropathology. 2015;35(5):469-480.

98. Markoullis K, Sargiannidou I, Gardner C, Hadjisavvas A, Reynolds R, Kleopa KA. Disruption of oligodendrocyte gap junctions in experimental autoimmune encephalomyelitis. Glia. 2012;60(7):1053-1066.

99. Markoullis K, et al. Gap junction pathology in multiple sclerosis lesions and normal-appearing white matter. Acta Neuropathol. 2012;123(6):873-886.

100.Parenti R, et al. Dynamic expression of Cx47 in mouse brain development and in the cuprizone model of myelin plasticity. Glia. 2010;58(13):1594-1609.

101.Cole KLH, Early JJ, Lyons DA. Drug discovery for remyelination and treatment of MS [published online ahead of print June 15, 2017]. Glia. https:// doi.org/10.1002/glia.23166.

102. Fan HB, et al. Transplanted miR-219-overexpressing oligodendrocyte precursor cells promoted remyelination and improved functional recovery in a chronic demyelinated model. Sci Rep. 2017;7:41407.
103. Robberecht W, Philips T. The changing scene of amyotrophic lateral sclerosis. Nat Rev Neurosci. 2013;14(4):248-264.

104. Ling SC, Polymenidou M, Cleveland DW. Converging mechanisms in ALS and FTD: disrupted RNA and protein homeostasis. Neuron. 2013;79(3):416-438.

105. Bensimon G, Lacomblez L, Meininger V. A controlled trial of riluzole in amyotrophic lateral sclerosis. ALS/Riluzole Study Group. N Engl J Med. 1994;330(9):585-591.

106.Sawada H. Clinical efficacy of edaravone for the treatment of amyotrophic lateral sclerosis. Expert Opin Pharmacother. 2017;18(7):735-738.

107. Kang SH, et al. Degeneration and impaired regeneration of gray matter oligodendrocytes in amyotrophic lateral sclerosis. Nat Neurosci. 2013;16(5):571-579.

108. Philips T, et al. Oligodendrocyte dysfunction in the pathogenesis of amyotrophic lateral sclerosis. Brain. 2013;136(pt 2):471-482.

109. Ferraiuolo L, et al. Oligodendrocytes contribute to motor neuron death in ALS via SOD1dependent mechanism. Proc Natl Acad Sci US A. 2016;113(42):E6496-E6505.

110. Cui Y, et al. Extensive dysregulations of oligodendrocytic and astrocytic connexins are associated with disease progression in an amyotrophic lateral sclerosis mouse model. J Neuroinflammation. 2014;11:42.

111. Almad AA, et al. Connexin 43 in astrocytes contributes to motor neuron toxicity in amyotrophic lateral sclerosis. Glia. 2016;64(7):1154-1169. 